\title{
Occupant-detection strategy using footstep-induced floor vibrations
}

\author{
Slah Drira \\ slah.drira@epfl.ch \\ Swiss Federal Institute of Technology \\ (EPFL) \\ Lausanne, Switzerland
}

\author{
Yves Reuland \\ Swiss Federal Institute of Technology \\ (EPFL) \\ Lausanne, Switzerland
}

\author{
Nils F.H. Olsen \\ Swiss Federal Institute of Technology \\ (EPFL) \\ Lausanne, Switzerland
}

\author{
Sai G.S. Pai \\ Future Cities Laboratory, ETH Zurich \\ Singapore \\ Swiss Federal Institute of Technology \\ (EPFL) \\ Lausanne, Switzerland
}

\author{
Ian F.C. Smith \\ Future Cities Laboratory, ETH Zurich \\ Singapore \\ Swiss Federal Institute of Technology \\ (EPFL) \\ Lausanne, Switzerland
}

\begin{abstract}
Identification of occupant presence and location inside buildings is essential to functional goals such as security, healthcare, and energy management. Floor-vibration measurements, induced by footstep impacts, provide a non-intrusive sensing method for occupant identification, unlike cameras and smartphones. Detecting the presence of an occupant is a necessary first step for occupant location identification. A challenge for occupant detection is ambient noise that may hide footstep-induced floor-vibration signatures. Also, spurious events such as door closing, chair dragging and falling objects may result in vibrations that have similarities with footstep-impact events. In this paper, an accurate occupant-detection strategy for structures with varying rigidity is outlined. Event detection is based on computing the standard deviation of a moving window over measurements at various frequency ranges. Using a classification method, footsteps are distinguished from other events. This strategy enhances detection of footstep-impact events compared with methods that employ only thresholds, thereby reducing false positives (incorrect detection) and false negatives (undetected events). Footstep-impact events may then be used for footstep impact localization using model-based approaches. Finally, the utility of this strategy for footstep-event detection is evaluated using a full-scale case study.
\end{abstract}

\section{CCS CONCEPTS}

- Computing methodologies $\rightarrow$ Support vector machines.

\section{KEYWORDS}

Occupant detection, Footstep-induced floor vibrations, Footstepimpact event, Classification method

Permission to make digital or hard copies of all or part of this work for personal or classroom use is granted without fee provided that copies are not made or distributed for profit or commercial advantage and that copies bear this notice and the full citation on the first page. Copyrights for components of this work owned by others than ACM must be honored. Abstracting with credit is permitted. To copy otherwise, or republish, to post on servers or to redistribute to lists, requires prior specific permission and/or a fee. Request permissions from permissions@acm.org.

DFHS 19, November 10, 2019, New York, NY, USA

(C) 2019 Association for Computing Machinery.

ACM ISBN 978-1-4503-7007-3/19/11.

https://doi.org/10.1145/3360773.3360881
ACM Reference Format:

Slah Drira, Yves Reuland, Nils F.H. Olsen, Sai G.S. Pai, and Ian F.C. Smith. 2019. Occupant-detection strategy using footstep-induced floor vibrations. In DFHS 19: ACM Workshop on Device-Free Human Sensing, November 10, 2019, New York, NY, USA. ACM, New York, NY, USA, 4 pages. https://doi. org $/ 10.1145 / 3360773.3360881$

\section{INTRODUCTION}

Occupant detection is a crucial step for locating occupant inside buildings. Information regarding flow of building occupants has the potential to understand occupant behavior. Thus, several building applications, such as security systems and energy management, may be optimized.

Several sensing technologies, such as embedded and portable sensors, have been used to identify the presence of occupants. Research on occupant detection has involved equipment such as optical sensors [6] and radio-frequency devices [8]. Due to the intrusive nature of these devices, unobtrusive strategies, such as vibration sensors, are often preferred since they preserve privacy. Occupant detection using vibration measurements involves footstep impacts that generate structural vibrations, which travel through the floor slab to be captured by vibration sensors.

Building floors present varying rigidities and obstructions such as beams and walls. This may explain the dispersive nature of real floor slabs, thus leading to footstep-impact events with low signalto-noise ratio (SNR) and vibration shapes that change according to sensor locations $[9,10]$. Threshold-based methods have been used for occupant detection [4,9]. A footstep-event is detected as an anomaly when vibration amplitudes exceed baseline level of ambient vibrations. However, a main challenge for occupant detection is ambient noise that may cover footstep-induced floorvibration signatures [7]. Also, spurious events such as door closing, chair dragging or falling objects might result in similar vibrations [9].

In order to overcome this limitation, signal resolution has been increased using sensor-based amplifier [9]. Also, a discrete-wavelettransform technique with high-level filtering has been used to denoise vibration measurements to differentiate impact events from 
ambient noise [3]. However, this might lead to filtering out footstepevent signals. Unsupervised learning techniques using Gaussianmixture models has been used to detect and extract events [1]. However, distinguishing footstep events from other impact sources has not been tested.

Supervised learning techniques based on one-class Support Vector Machine (SVM), which maximizes the distance between data points and the separating hyperplane [2] have been used to classify footstep events from other spurious impulses [3, 7]. The motivation behind the use of one-class SVM is to train the learning process with only positive events. However, such training may miss-classify spurious impulses as footstep events.

In this paper, an accurate event-detection strategy for building structures is outlined (Section 2). Various frequency components of vibration measurements are explored to differentiate between impact events. Event detection is based on computing the standard deviation of a moving window over several frequency components of the measurements. Local maxima resulting from standard deviation values correspond to possible impact events. Then a classification method using a binary SVM is performed to distinguish footstep events from other events (Section 3). Application to a full-scale system is presented in Section 4 and conclusions are discussed in Section 5 .

\section{EVENT DETECTION}

Event detection from vibration measurements is a necessary first operation for identification of occupant location. Impacts generate non-stationary waves that travel through the slab with phase velocities that depend on frequencies [9]. These waves are affected by natural modes of the structure. Thus, this event-detection strategy is based on exploring information from frequency components of floor-vibration measurements. Figure 1 describes the eventdetection strategy.

The measured signal is firstly decomposed using a continuous wavelet transform (CWT) at frequency ranges that contain the fundamental vertical modes of the structure. This enhances the detection of events with low SNR that may be differentiable from ambient noise at either low or high-frequency ranges. The Morlet wavelet is used as mother wavelet due to its shape similarity with footstep-impact signals.

Based on experimental studies, it has been found that the walking frequency varies between $1.4 \mathrm{~Hz}$ and $2.5 \mathrm{~Hz}$ [11]. Thus, each decomposed signal is segmented using a moving window of $0.2 \mathrm{~s}$ with an overlap of $0.1 \mathrm{~s}$. The duration of the moving window corresponds to half of the minimum walking duration (equal to $0.4 \mathrm{~s}$ ) $\left(D_{\text {walk, } \min }\right)$ to cover all possible stepping frequencies. Maximum standard deviation of all sensors $\left(S T D_{\max }\right)$ is computed for each signal segment (SS) and repeated for all decomposed signals. Detection thresholds are computed based on $S T D_{\max }$ of ambient noise that is decomposed at same frequency ranges. $S T D_{\max }$ values are taken to be detection thresholds (see dashed lines in Figure 2).

Local maxima resulting from $S T D_{\max }$ values (that exceed detection threshold) over each decomposed signal correspond to possible events (see Figure 2). Each local maximum is picked when the corresponding SS has greater $S T D_{\max }$ value within an interval of $D_{\text {walk, min. }}$. However, each decomposed signal may result in local maxima that correspond to a same impact event. Also, varying amplitudes in an event signal may lead to detecting several local maxima. In order to remove local maxima that correspond to a same impact event, an assumption of an occupant walks with relatively constant walking frequency is made. Thus, an approximate walking frequency $\left(f_{\text {walk }}\right)$ is computed based on the first ten detected local maxima. If the $f_{\text {walk }}$ is lower than $2 \mathrm{~Hz}$, information from each decomposed signal is combined by taking the first local maximum that exceeds the $D_{\text {walk, min }}$ from the previously detected event. Otherwise, the first local maximum that exceeds half of the $D_{\text {walk, min }}$ from the previously detected event presents the next impact event.

SSs of the resulting local maxima are used as baseline to extract impact-event signals at each sensor location. Each SS that represents an impact event is used with the time that corresponds to maximum amplitude of all sensors. This allows the definition of two windows of durations of $0.15 \mathrm{~s}$ before and $0.45 \mathrm{~s}$ after this time. The total duration of $0.6 \mathrm{~s}$ is the average walking period. These two windows are used to identify the starting and the ending duration of each event signal as well as the minimum peak value of all sensors.

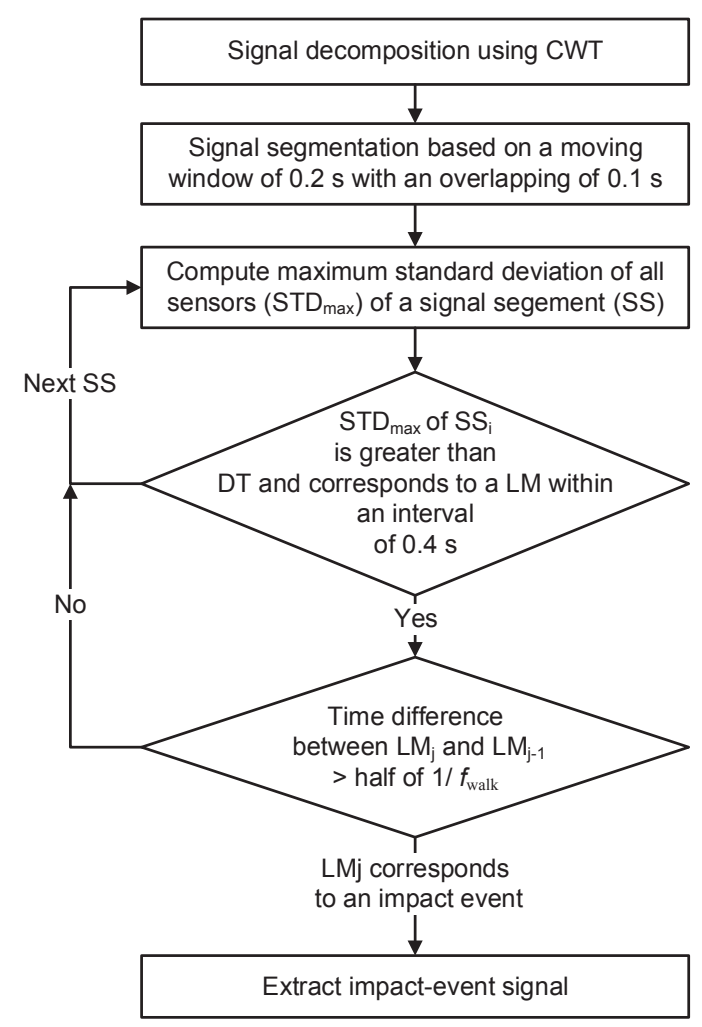

Figure 1: Event-detection strategy is explained. Measured signal is decomposed using CWT at frequency ranges that contain first natural modes of the structure. $S S_{i}$ is signal segment of a running window i. $D T$ is detection threshold. $L M_{i}$ is the last detected local maximum. $L M$ resulting from each decomposed signal are combined based on the $f_{\text {walk }}$. 


\section{EVENT CLASSIFICATION}

Real-life applications for occupant identification using floor-vibration measurements are often complicated by spurious events such as door closing, chair dragging or falling objects. These events might result in similar vibrations than footstep-impact signals. Thus, a supervised learning technique based on binary-SVM is used to classify footstep events from other impulses. SVM is used to find the optimal decision boundary that maximizes the distance between data points and the separating hyperplane. A Gaussian kernel is used to perform a non-linear separation between footstep and nonfootstep classes. Data sets that include footstep and non-footstep events are then used to train the binary-SVM classifier.

Feature extraction is essential for event classification to differentiate footstep event from other impulses. Natural vibration modes influence the response of structures to impacts and thus, features are assessed in time and frequency domains. Each event signal is decomposed using CWT and recomposed at equivalent frequency intervals with a range of $20 \mathrm{~Hz}$ between $10 \mathrm{~Hz}$ and $260 \mathrm{~Hz}$. STD, maximum difference in amplitudes, root-mean-square, kurtosis, median and entropy of an event signal are computed at each sensor location and for each frequency range. Maximum and mean values over all sensors are taken for all metrics. In addition, frequency that corresponds to maximum of first singular values of cross-powerspectral density (CPSD) over all sensors and the centroid of first singular values of CPSD are calculated. These metrics are used as candidate metrics for event-classification feature selection.

\section{APPLICATION TO FULL-SCALE FLOOR SLABS}

The occupant-detection strategy is applied to two full-scale studies. The first case study is a reinforced-concrete slab (approximately $950 \mathrm{~m} 2$ ) located in Singapore [5]. The second case study is an office room located in Switzerland. The floor is a continuous reinforcedconcrete slab (approximately $90 \mathrm{~m}^{2}$ ). The slab is $20 \mathrm{~cm}$ thick and supported by six steel columns. A non-structural wall made of plasterboard and masonry walls separate the office room. Eight sensors (Geophones SM-24 by I/O Sensor Nederland bv) are used to measure vertical velocity responses of the two case-studies at a sampling rate of $1000 \mathrm{~Hz}$.

Prior analysis of ambient-vibration measurements has revealed that dominant vertical modes of the first case study are in the range of [9-15] Hz. Whereas the fundamental vertical bending modes of the second case study are found in the frequency range of [20-25] $\mathrm{Hz}$.

\subsection{Event-detection results}

Measurements are decomposed using CWT at frequency ranges that contain first natural modes of the structure (see Section 2). Figure 2 presents an example of event detection of four footstep-events for the first case study. STD max of a moving window is computed for the decomposed vibration signal at four frequency ranges: [515] Hz (see Figure 2b), [10-20] Hz, [15-25] Hz and [20-30] Hz (see Figure 2c). Dashed lines are detection thresholds. Triangles are the detected events. Figure $2 \mathrm{~b}$ shows that the decomposed signal at the first bending mode of the structure is not able to detect the two first footstep events since they are below the detection thresholds.
Table 1: Footstep-event detection results

\begin{tabular}{lcl}
\hline & Detected & Non-detected \\
\hline BBR building Singapore & 2584 & 9 \\
Office room Switzerland & 1851 & 5 \\
\hline
\end{tabular}

However, events with low SNR are differentiable from ambient noise (decomposed at same frequency range) in higher frequency components, as illustrated in Figure 2c. Combining information from decomposed signals at several frequency ranges reduces false negatives.
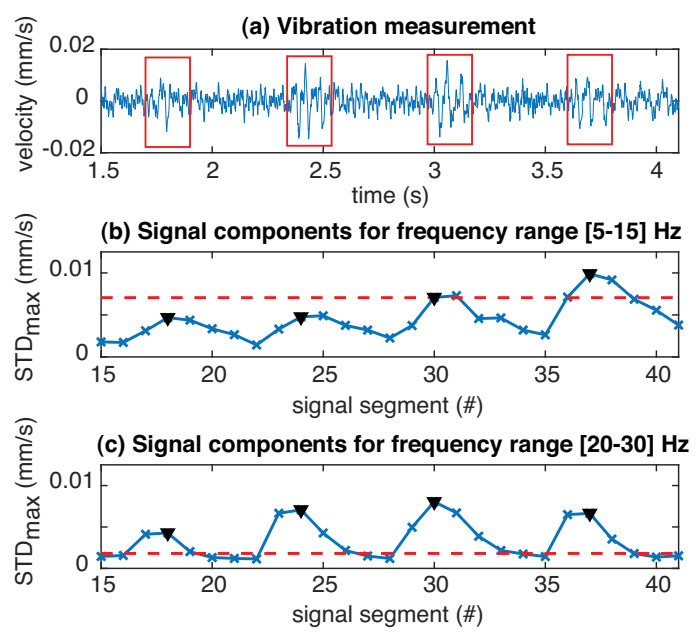

\begin{tabular}{|cl|}
\hline$-\bar{x}$ & Detection thresholds \\
$\nabla$ & STD $\operatorname{\text {max}}$ values \\
$\square$ & Detected maxima that correspond to impact events \\
\hline
\end{tabular}

Figure 2: (a) presents an example of vibration measurements of a walking occupant in the first case study. (b and c) presents $S T D_{\max }$ values of the vibration signal that is decomposed for frequency ranges of [5-15] $\mathrm{Hz}$ and [20-30] Hz. Triangles are local maxima that correspond to detected impact events.

More than 4400 footstep signals have been recorded on the two case studies to test the event-detection strategy. Measurements include various occupants with non-fixed walking frequency and multiple shoe types. Table 1 presents the number of detected footstep events for each case study, where more than $99 \%$ of all footstep events are successfully detected. In addition, no more than two false negatives were present within a single trajectory measurement of a walking person.

\subsection{Event-classification results}

Measurements have been carried out for five people walking along a fixed trajectory in the second case study. In addition, book-dropping, chair-dragging, table-hitting and opening/closing-door events have been recorded. Blind tests including bounces of a ball, hand and mug impacts on a table, chair-dragging, opening/closing-door as well as occupant walking and jumping events are tested for predictions. 
Table 2: Classification performance based on validation test and blind tests

\begin{tabular}{ccl}
\hline & Validation Test & Blind Tests \\
\hline Recall & 99.3 & 78.1 \\
Precision & 99.3 & 95 \\
Accuracy & 98.9 & 81.3 \\
F1 Score & 99.3 & 85.7 \\
\hline
\end{tabular}

The data set for binary-SVM classification includes 1851 footstep events and 395 non-footstep events. The blind tests include 161 footstep events and 111 non-footstep events that are used to estimate the prediction performance. The data set is split into $75 \%$ for training and $25 \%$ for validation.

The key of event classification is to select good features. Event metrics that maximize the discrepancy between footstep and nonfootstep event clusters are selected as features for classification. Null-hypothesis tests to all frequency ranges of each metric have been conducted to select the frequency range that significantly differentiates footsteps from other events. Also, highly correlated metrics are avoided. Features that are found to be useful in separating footsteps from other events are: maximum STD of all sensors of event signals at frequency range of [60-80] Hz, maximum kurtosis of all sensors of event signals at frequency range of [10-30] Hz, spectral centroid and frequency that corresponds to maximum first singular values of CPSD.

In Table 2, the performance of the binary-SVM classifier is presented using four metrics: recall, precision, accuracy and F1 score. Recall gives the proportion of the number of detected true-footstep events from all true-footstep events. Precision presents the proportion of the number of detected true-footstep events from all events classified as footstep events. Accuracy reflects the number of correct predictions divided by the total number of predictions. F1 score is the overall performance metric on the classifier model's ability to distinguish between footstep and non-footstep events. The binarySVM classifier leads to high performance scores (average of $99 \%$ ) for validation test. However, lower prediction-performance scores (average of $86 \%$ ) are obtained for the blind tests.

Table 3 illustrates the prediction accuracy of the binary-SVM classifier of several events (that are not included in the training data set) such as bounces of a ball, jumping, chair-dragging and footstep events of three occupants. Binary-SVM classifier predicts footstep events with an average of $96 \%$. Also, the classifier accurately differentiates chair-dragging events from stepping events. However, jumping and bounces of a ball are classified with an accuracy of $60 \%$ and $42 \%$ respectively. This may explain the reduction of the overall performance of the blind-test prediction compared with the validation test (see Table 2). The classifier does not predict well events that are not included in the training set. Exploring new features and extending the training set to include additional non-footstep events may increase the prediction-performance scores.

\section{CONCLUSIONS}

An occupant-detection strategy has been developed and applied to two building slabs. The following conclusions are drawn:
Table 3: Prediction accuracy of several events from the blind tests

\begin{tabular}{ccl}
\hline & Event \# & Accuracy (\%) \\
\hline Chair dragging Footsteps (AT) & 2427 & 94.3 \\
Bounces of a ball & 55 & 41.8 \\
Footsteps (SP) & 58 & 98.3 \\
Footsteps (YR) & 76 & 94.7 \\
Jumping & 20 & 60 \\
\hline
\end{tabular}

- Combining information from multiple frequency components of measurement vibration leads to accurate eventdetection.

- Low-and-high frequency components in feature selection increases the classification performance.

Future work includes testing additional features and adding training data in order to enhance event classification.

\section{ACKNOWLEDGMENTS}

This work was funded by the applied computing and mechanics laboratory EPFL, the Singapore-ETH centre (SEC) under contract no. FI 370074011-370074016 and the Swiss National Science Foundation (SNSF) under contract no. 200020-169026.

\section{REFERENCES}

[1] Sahil Anchal, Bodhibrata Mukhopadhyay, and Subrat Kar. 2017. UREDT: Unsupervised learning based Real-Time footfall event detection technique in seismic signal. IEEE sensors letters 2, 1 (2017), 1-4.

[2] Rezaul K Begg, Marimuthu Palaniswami, and Brendan Owen. 2005. Support vector machines for automated gait classification. IEEE transactions on Biomedical Engineering 52, 5 (2005), 828-838.

[3] Jose Clemente, Fangyu Li, Maria Valero, and WenZhan Song. 2019. Smart seismic sensing for indoor fall detection, location and notification. IEEE journal of biomedical and health informatics (2019).

[4] Slah Drira, Yves Reuland, Sai G S Pai, Hae Young Noh, and Ian F C Smith. 2019. Model-Based Occupant Tracking Using Slab-Vibration Measurements. Frontiers in Built Environment 5 (may 2019), 63. https://doi.org/10.3389/fbuil.2019.00063

[5] Slah Drira, Yves Reuland, and Ian F C Smith. 2019. Model-based interpretation of floor vibrations for indoor occupant tracking. In 26th International Workshop On Intelligent Computing In Engineering. Leuven Belgium.

[6] Varick L Erickson, Stefan Achleitner, and Alberto E Cerpa. 2013. POEM: Powerefficient occupancy-based energy management system. In Proceedings of the 12 th international conference on Information processing in sensor networks. ACM, Philadelphia, Pennsylvania, USA, 203-216.

[7] Mike Lam, Mostafa Mirshekari, Shijia Pan, Pei Zhang, and Hae Young Noh. 2016. Robust occupant detection through step-induced floor vibration by incorporating structural characteristics. In Dynamics of Coupled Structures, Volume 4. Springer, 357-367.

[8] Patrick Lazik, Niranjini Rajagopal, Oliver Shih, Bruno Sinopoli, and Anthony Rowe. 2015. ALPS: A bluetooth and ultrasound platform for mapping and localization. In Proceedings of the 13th ACM conference on embedded networked sensor systems. ACM, Seoul, South Korea, 73-84.

[9] Mostafa Mirshekari, Shijia Pan, Jonathon Fagert, Eve M Schooler, Pei Zhang, and Hae Young Noh. 2018. Occupant localization using footstep-induced structural vibration. Mechanical Systems and Signal Processing 112 (2018), 77-97.

[10] Shijia Pan, Tong Yu, Mostafa Mirshekari, Jonathon Fagert, Amelie Bonde, Ole J Mengshoel, Hae Young Noh, and Pei Zhang. 2017. Footprintid: Indoor pedestrian identification through ambient structural vibration sensing. Proceedings of the ACM on Interactive, Mobile, Wearable and Ubiquitous Technologies 1, 3 (2017), 89.

[11] Vitomir Racic, Aleksandar Pavic, and J M W Brownjohn. 2009. Experimental identification and analytical modelling of human walking forces: Literature review. Journal of Sound and Vibration 326, 1-2 (2009), 1-49. 